\title{
Numerical Simulation of Blood Flow in a Vessel by Using COMSOL Multiphysics ${ }^{\circledR}$ Software
}

\author{
S. F. Salem ${ }^{1,2^{*}}$ and V. V. Tuchin ${ }^{1,3,4,5}$ \\ ${ }^{1}$ Department of Optics and Biophotonics, Saratov State University, Saratov 410012, Russia. \\ ${ }^{2}$ Department of Physics, Faculty of Science, Benha University, Egypt. \\ ${ }^{3}$ Interdisciplinary Laboratory of Biophotonics, Tomsk State University, Tomsk 634050, Russia. \\ ${ }^{4}$ Laboratory of Molecular Imaging, Bach Institute of Biochemistry, Research Center of Biotechnology \\ of the Russian Academy of Sciences, Moscow 119071, Russia. \\ ${ }^{5}$ Institute of Precision Mechanics and Control of the Russian Academy of Sciences, \\ Saratov 410028, Russia.
}

\section{Authors' contributions}

This work was carried out in collaboration between both authors. Author SS designed the study, performed the modelling and analysis and wrote the first draft of the manuscript. Author VT managed the analyses of the modelling results and the literature search. Both authors read and approved the final manuscript.

Article Information

DOI: $10.9734 / A R R B / 2020 / v 35 i 930272$ Editor(s):

(1) Dr. David E. Martin, Martin Pharma Consulting, LLC \& DFH Pharma Inc., USA. (2) Dr. Gonzalo Emiliano Aranda Abreu, Veracruzana University, Mexico. (3) Dr. Paola Angelini, University of Perugia, Italy. Reviewers:

(1) Shailesh Mishra, Dr. A. P. J. Abdul Kalam Technical University (AKTU), India. (2) Ashraf El Droubi, University of São Paulo (USP), Brazil. (3) Gustavo Boroni, Universidad Nacional del Centro de la Provincia de Buenos Aires (UNCPBA), Argentina. Complete Peer review History: http://www.sdiarticle4.com/review-history/60148

Original Research Article

Received 18 June 2020

Accepted 23 August 2020

Published 02 September 2020

\section{ABSTRACT}

Dynamics of the blood flow play an important role in the development and treatment of cardiovascular diseases. In recent decades, blood flow simulation has been widely used to better understand the symptomatic spectrum of different diseases, in order to improve existing or develop new therapeutic techniques. Numerical simulation for biomagnetic fluid (such as blood) flow through a tube with rectangular cross section under the influence of magnetic field is studied in this work. Blood considered as a magnetic and incompressible fluid. The magnetic field effects on the blood stream in a tube are created by a permanent magnet outside the tube. The equations of motion 
which describe the flow is governed by the combination of magnetic equations for permanent magnet and Navier-Stokes equation for fluid (blood) were solved numerically by using COMSOL Multiphysics $®$ Modeling Software.

Keywords: Magnetism; Newtonian fluid; non-Newtonian fluid; permanent magnet; computational modeling.

\section{INTRODUCTION}

Biological fluid dynamics (such as blood or lymph) in the presence of a magnetic field can play an important role in many bioengineering and biomedical applications [1-11]. In this regard, numerous studies have been carried out over the past decades that in most cases, the movement of Newtonian, non-Newtonian biofluids and numerical simulation for magnetic fluid in rectangular and circular tubes are described [1217]. Blood behaves as a magnetic fluid due to the dynamic interaction with magnetic field between the intercellular proteins, cell membrane, and hemoglobin. The magnetic property of blood is determined by the hemoglobin oxygenation state (a form of iron oxide in red blood cells (RBCs)) [18-21]. When blood is pumped through the tube, at the high magnetic effect of the external magnetic field, the magnetic particles (RBCs or special magnetic drug delivery containers) are directed towards the tube wall and accumulated by the wall. Blood magnetization can be considered as a paramagnetic material in this region where deoxygenated blood is flowing. When the magnet is removed, the magnetic field effect vanishes and blood acts as a homogeneous fluid and its velocity profile is parabolic.

Tzirtzilakis et al. [12] suggested a mathematical model of biological fluid dynamics (BFD) and was studying an application where numerically studied the three-dimensional, fully developed, viscous flow of a biomagnetic fluid under the influence of a magnetic field. Numerical results are obtained by using an efficient technique based on a pseudotransient, pressure-linked system implemented on a standard orthogonal grid. In general, the results showed that the magnetic field reduces the rate of flow.

Tzirtzilakis et al. [13], in another work studied numerically the movement of BFD in a channel with symmetric stenosis. The numerical solution of the problem is based on the numerical finite difference developed. Results concerning the velocity field show that the symmetry of the flow downstream of the stenosis breaks and the vortex near the source of the magnetic field is enlarged.

Tzirakis et al. [14]. Presented a mathematical model for the explanation of the exposed biomagnetic fluid flow to a magnetic field that accounts for both the biofluid's electrical and magnetic properties. It is achieved by applying the forces of Lorentz and magnetization to the Navier-Stokes equations, and considered the case of laminar, incompressible, viscous, a steady flow of Newtonian biomagnetic fluid (i) between two parallel plates; and (ii) through a straight rigid tube 60 percent in diameter, 84 percent in area, axisymmetric stenosis. Two external magnetic fields were examined: one produced by an infinite wire carrying constant current, and a field similar to a dipole. They demonstrated, numerically and analytically, that the wire creates an irrotational force that, regardless of its strength, alters only the pressure leaving the field of velocity unimpacted. By contrast, if the fluid is exposed to the dipolelike field, which causes rotational force, then pressure and velocity can be strongly affected even at moderate field strengths.

Misra and Shit [15], examined various aspects of blood flow in various segments of the circulatory system, in a situation where the system was subjected to an external magnetic field. Their analysis showed that the instantaneous flow characteristics are significantly influenced by the magnetic parameter and the instability parameter as well as the phase angle of the radius.

Probst et al. [16]. Considered the initial problem of ferro- fluid control; the precise manipulation of a single drop of ferro- fluid by four external electromagnets; the location of the droplet was sensed by a camera and imaging software, and the electromagnets were then correctly actuated at each time to move it from where it should be closer to where it should be.

Alexandru et al. [17]. Provided the mathematical model and the findings of numerical simulations for complex arterial blood flow-structural coupled models unique to the targeting of magnetic drugs 
(MDT). The computational domains are created in their approach using image-based reconstruction techniques, providing a more realistic definition.

As blood is pumped into the vessel, the magnetic particles (RBCs, magnetic nanoparticles, or special magnetic drug delivery containers) are guided towards the vessel's wall and collected close to the wall at the external magnet's high magnetic field. Blood magnetization can be regarded as a paramagnetic substance in the area where deoxygenated blood flows when the magnet is removed, the effect of magnetic field vanishes and blood behaves as a homogenous fluid with a parabolic velocity profile.

In this work, blood is considered as Newtonian and non-Newtonian fluid. The equations of motion describing the flow by the combination of magnetic equations for permanent magnet and Navier-Stokes equation for fluid (blood) were solved numerically by using COMSOL Multiphysics $®$ Modeling Software.

\section{FORMULATION OF THE PROBLEM}

Biomagnetic fluid (blood) flow in a tube with the rectangular cross-section.is analyzed. In this model, blood flow considered as a laminar steady flow of viscous and incompressible fluid. The motion of blood is considered in two dimensions $(\mathrm{x}, \mathrm{y})$ with the corresponding velocity components in Cartesian coordinates (u, v). The magnetic field which produced by a permanent magnet is located outside from the tube. The model domain for solution of the problem contains two domains: first, the tube domain containing biomagnetic fluid such as blood, and the second domain of a permanent magnet and the surrounding medium is air as it is shown in( Fig.1A).

The COMSOL Multiphysics ${ }^{\circledR}$ Software was used to solve equations for flow and magnetic field numerically under the initial and boundary conditions based on a finite element method.

\section{EQUATIONS OF MOTION AND RESULTS}

\subsection{Used Modules of the Comsol Multiphysics $®$ Software}

To provide calculations, the equations describing the problem were solved numerically by using two different modules of the COMSOL Multiphysics $®$ Software. These modules include:

1. AC/DC module to calculate the magnetic field of the permanent magnet.

2. CFD module for laminar fluid flow such as blood. The flow of blood in this problem is described by Navier-Stokes equation, which considers blood as a Newtonian and non- Newtonian fluid.

\subsection{Magnetic Field}

A stationary magnetic field produced by a permanent magnet implanted at a specific location is described by the magnetostatic equations for the static magnetic field derivered from the Ampere-Maxwell equation [22]:

$$
\nabla \times \overline{\mathrm{H}}=\overline{\mathrm{J}}
$$

Gauss law for magnetic flux density is given by:

$$
\nabla \cdot \overline{\mathrm{B}}=0,
$$

and the magnetic flux density in different domains can be described by the relation between $\overline{\mathrm{B}} \& \overline{\mathrm{H}}$, which is given by theses formulas:

for the blood stream

$$
\overline{\mathrm{B}}=\mu_{0}\left(\overline{\mathrm{H}}+\overline{\mathrm{M}_{\mathrm{b}}}(\mathrm{H})\right)
$$

for the permanent magnet

$$
\overline{\mathrm{B}}=\mu_{0} \mu_{\mathrm{r}} \overline{\mathrm{H}}+\mathrm{B}_{\mathrm{rem}},
$$

for the air

$$
\overline{\mathrm{B}}=\mu_{0} \overline{\mathrm{H}}
$$

where $\mu_{0}$ is the magnetic permeability of air and it is constant $\mu_{0}=4 \pi \times 10^{-7} \mathrm{~N} / \mathrm{A}^{2} ; \mu_{\mathrm{r}}$, is the relative magnetic permeability of the permanent magnet; $\mathrm{H}$ is the magnetic field strength; $\mathrm{B}$ is the magnetic flux density; $B_{\text {rem }}$ is the remanent magnetic flux density; $M_{b}(H)$ is the magnetization vector of the blood stream $(\mathrm{A} / \mathrm{m})$, which is a function of magnetic field, $\mathrm{H}$. 


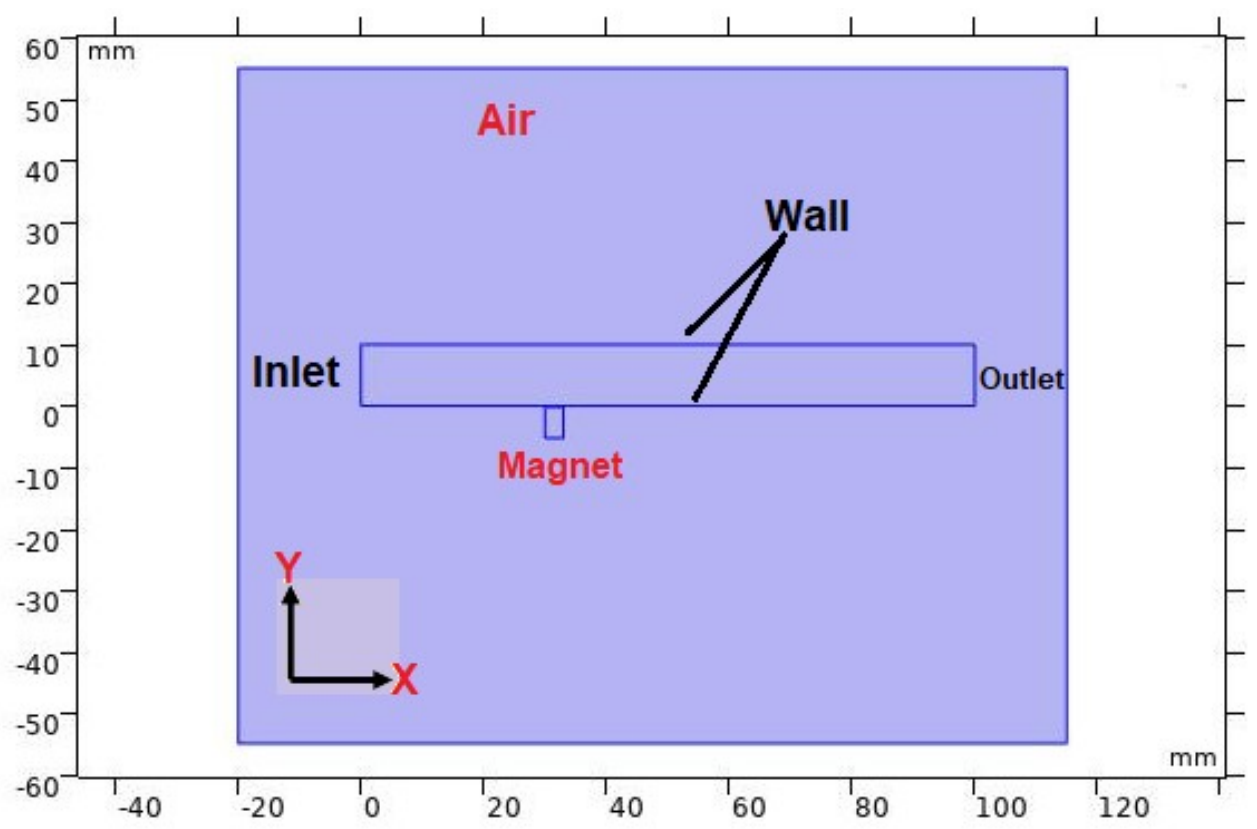

A

Fig. $1 \mathrm{~A}$. Geometric domains for the model. The tube domain is rectangular cross section with dimensions $100 \mathrm{~mm} \times 10 \mathrm{~mm}$, the permanent magnet with dimensions $3 \mathrm{~mm} \times 5 \mathrm{~mm}$ (A)

\subsection{Equations of Motion for the Fluid (Blood)}

The motion of blood through the tube can be expressed by incompressible Navier-Stokes equations [23-25]:

$$
\rho \frac{\partial \overline{\mathrm{u}}}{\partial \mathrm{t}}+\rho(\overline{\mathrm{u}} \cdot \nabla) \overline{\mathrm{u}}=-\nabla \mathrm{P}+\eta \nabla^{2} \overline{\mathrm{u}}+\overline{\mathrm{F}},
$$

where $\overline{\mathrm{u}}$ is the velocity vector; $\rho$ is the density; $\nabla \mathrm{p}$ is the pressure gradient; $\eta$ is the blood dynamic viscosity; $\overline{\mathrm{F}}$ is the external force per unit volume, it includes any external forces such as gravity or electromagnetic force, in this work, the force term represents the magnetic volume force, which is acting on blood stream.

For non - Newtonian blood flow, the CarreauYasuda model $[26,27]$ for the shear thinning behaviour of blood is also commonly used in hemodynamical simulations. The viscosity is given by Eq.(7):

$$
\eta\left(\gamma^{*}\right)=\eta_{\infty}+\left(\eta_{0}-\eta_{\infty}\right)\left[1+\left(\lambda \gamma^{*}\right)^{2}\right]^{\mathrm{n}-1}
$$

where $\lambda$ and $\mathrm{n}$ constant parameters are dimensionless.

\subsection{Boundary Conditions for the Fluid (Blood)}

The blood flow was considered to be a steady flow, which was supposed to flow into the tube from the inlet and to exit the tube in the outlet. Thus, velocity was applied for the inlet section and a fixed pressure for the outlets. At the inlet of the tube, the velocity profile for blood in $x$-axis and the flow with zero velocity in $y$ - direction. No slip condition for all tube walls was assumed, i.e. $(u=0)$ as in ( Fig. 1,A).

The inlet velocity described by $\left[u_{x}=u_{\max }(1-\right.$ $\left.\left.(x / h)^{2}\right)\right], u_{\max }$ is the maximum velocity, $h$ is the tube height, the density of blood $=1060 \mathrm{Kg} / \mathrm{m}^{3}$ and the dynamic viscosity is $\eta$ and it is constant for Newtonian fluid and for non-Newtonian fluid changes and depend on shear rate $\gamma^{\bullet}$ as shown in Eq.(7).

\section{DISCUSSIONS FOR RESULT}

In this work, the permanent magnet with magnetic field was applied to the tube with 
results presented in Fig. 3. From simulations, it follows that the greatest magnetic field strength is created in the vicinity to the tube wall surface.

The description of blood motion was done with the help of Navier-Stokes equation (6). For Newtonian flow of blood through the tube, surface of blood velocity distribution along the tube, the behavior for velocity profile parabolic flow for laminar flow i.e. velocity magnitude is minimal near the tube wall and maximal in the center, is shown in (Fig. 2B1).For non-Newtonian blood flow with dynamic viscosity dependent on shear rate as Eq. (7), the behavior of velocity more blunted, is shown in (Fig. 2C). The apparent viscosity decreases by increasing shear rate as (Fig. 2C1).

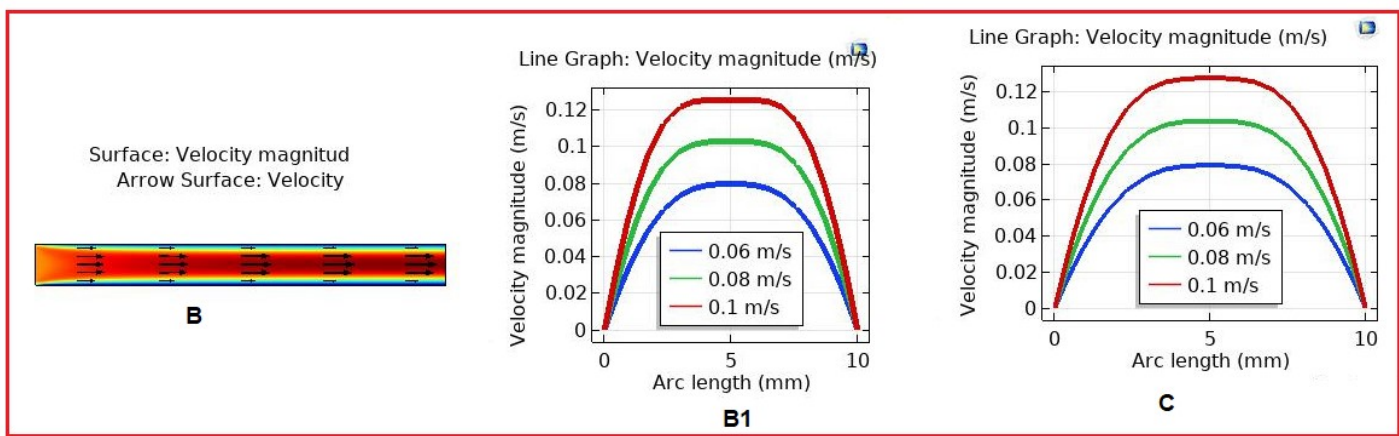

B1

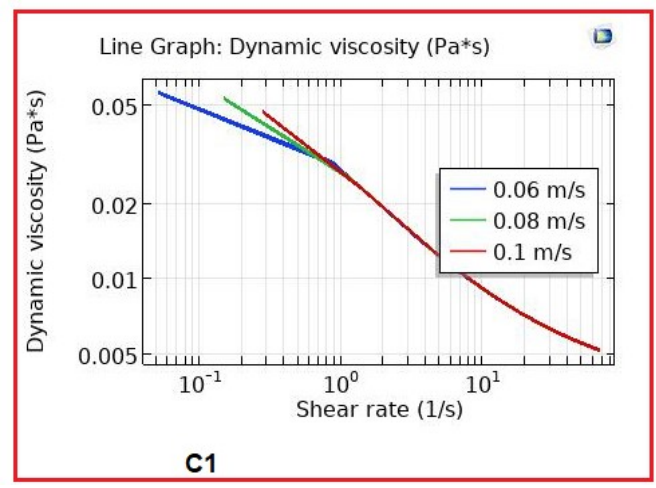

Fig. 2. Results of simulation: Surface of velocity magnitude for Newtonian blood flow in the tube B; line graph velocity profile for Newtonian and non-Newtonian blood flow inside the tube B1, C respectively; log scale Dynamic viscosity for non-Newtonian blood according to the formula of viscosity in Eq. (7) C1. Input velocity parametric sweep velocity values from $0.06 \mathrm{~m} / \mathrm{s}$ to $0.1 \mathrm{~m} / \mathrm{s}$

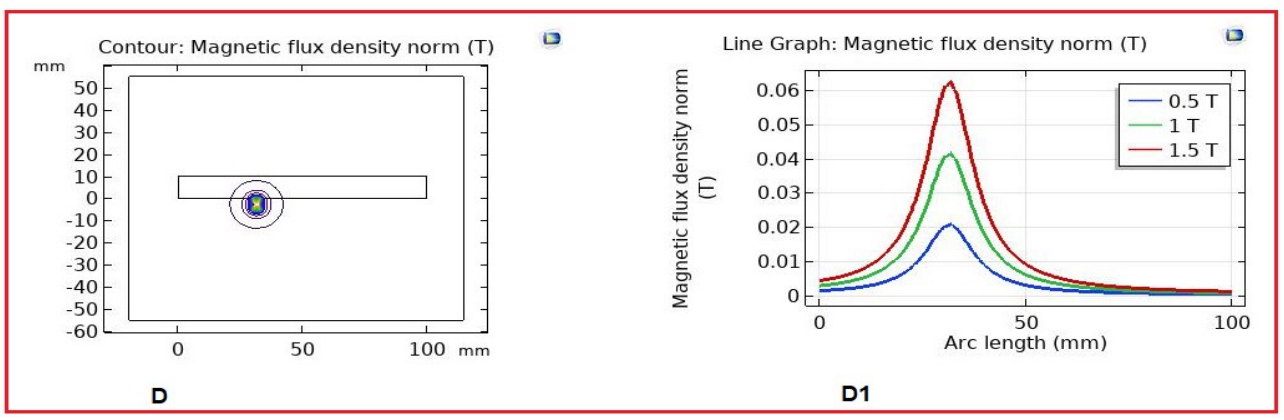

Fig. 3. Results of simulation: Contour of magnetic flux density for permanent magnet at a limit distance $x=30 \mathrm{~mm}$ as $D$; magnetic flux density along the center of tube (Arc length) for Parametric sweep from remnant magnetic flux densities as D1 


\section{CONCLUSION}

In this study, the magnetic field which is created by a permanent magnet located outside the tube is calculated. In this model, the fluid (blood) considered as a Newtonian and non-Newtonian fluid. The equations of motions, which are describing the flow through a tube, are governed by Navier Stokes equation for a fluid and magnetic equations for permanent magnet. They were solved numerically by using the COMSOL Multiphysics ${ }^{\circledR} \quad$ Modeling Software. Using simulations in biomechanics and applications of biomedicine are very important study in the recent decades [1-10]. The results of this study can be used in many biomedical applications such as using magnetic nanoparticles in drug delivery and drug targeting as shown in my previous work [28].

Other examples of the applications are treatment of cardiovascular conditions, such as stenosis and thrombosis. Thus, it is important to model not only the blood circulation but also the deformation of the blood vessel, In particular, it is important to have an accurate model of the interaction between the three components for optimizing the shape, size, and magnetic power, to efficiently deliver the drugs in the desired place and minimize side effects.

One of the future directions, the computational models used two-dimensional models for simplicity in this study, but our models can of course be expanded to three dimensions. The need for more computational resources emerges with increasing the size of the problem. Intensive research is under way in the area of domain decomposition, which helps to explain how to solve coupled multiphysics problems efficiently.

\section{DISCLAIMER}

The products used for this research are commonly and predominantly use products in our area of research and country. There is absolutely no conflict of interest between the authors and producers of the products because we do not intend to use these products as an avenue for any litigation but for the advancement of knowledge. Also, the research was not funded by the producing company rather it was funded by personal efforts of the authors.

\section{ACKNOWLEDGEMENT}

Authors are thankful to Maxim Kurochkin for the valuable discussions.

\section{COMPETING INTERESTS}

Authors have declared that no competing interests exist.

\section{REFERENCES}

1. Zhang X, Luo M, Tan P, Zheng L, Shu C. Magnetic nanoparticle drug targeting to patient-specific atherosclerosis: Effects of magnetic field intensity and configuration'. Applied Mathematics and Mechanics. 2020; 41(2):349-360.

Available:https://doi.org/10.1007/s10483020-2566-9

2. Bruno G, Vergara C. Computational comparison between Newtonian and nonNewtonian blood rheologies in stenotic vessels". Biomedical Technology, Springer, Cham. 2018;169-183.

3. Ismail F, Tamagawa M, Fazli Abdul Azi A, Wiriadidjaya S, Azrif Basri A, Arifin Ahmad K. Computational fluid dynamics study of blood flow in aorta using open FOAM. Journal of Advanced Research in Fluid Mechanics and Thermal Sciences. 2018;43: 81-89.

4. Mohammed Abdul KS, Raghuvir Pai AA, Arifin K, Ahmad Z, Prakashini K. Haemodynamics study in subject-specific abdominal aorta with renal bifurcation using CFD-A case study'. Journal of Advanced Research in Fluid Mechanics and Thermal Sciences. 2018;50(2):118-12.

5. Johny C, Pai R, Zuber M, Ahmad Z. Numerical study of haemodynamics behaviour in normal and single stenosed renal artery using fluid-structure interaction'. Journal of Advanced Research in Fluid Mechanics and Thermal Sciences. 2018;51(1):91-98.

6. Hamdipoor V, Afzal MR, Yoon J, Hapticbased manipulation scheme of magnetic nanoparticles in a multi-branch blood vessel for targeted drug delivery; 2018.

7. Rukshin I, Mohrenweiser J, Yue P, Afkhami S. 'Modeling superparamagnetic particles in blood flow for applications in magnetic drug targeting'. Fluids; 2017.

DOI: $10.3390 /$ fluids2020029

8. Alias NB, Ali A, Taha A, Abdul Hannan S, Aysh Dahwi A. 'Finite element simulation, characterization and transportation of magnetic nanoparticles under the impact of magnetic field in blood vessels'. Global Journal of Pure and Applied Mathematics. 2017;13(11):7771-7784. 
9. Voronin D, Sindeeva O, Kurochkin $M$, Mayorova O, Fedosov I, SemyachkinaGlushkovskaya O, Gorin D, Tuchin V, Sukhorukov G. In vitro and in vivo visualization and trapping of fluorescent magnetic microcapsules in a blood stream. ACS Applied Materials \& Interfaces. 2017; 9(8):6885-6893.

10. Gonzaga S, Oliveira C, Simões A. Computational fluid dynamics in abdominal aorta bifurcation: Non-Newtonian versus Newtonian blood flow in a real case study. Computer Methods in Biomechanics and Biomedical Engineering. 2017;20:822-831.

11. Nakagawa $H$, Ohuchi M. Virtual blood-flow controlling system: Optimization of human bioactivity under exposure to magnetic fields. IEEE Trans. Magn. 2016;52(7): 5000904

12. Tzirtzilakis EE, Sakalis VD, Kafoussias NG, Hatzikonstantinou PM. Biomagnetic fluid flow in a 3D rectangular duct.' International Journal for Numerical Methods in Fluids. 2004;44(12):1279-1298.

13. Tzirtzilakis EE. Biomagnetic fluid flow in a channel with stenosis. Physica D. 2008; 237:66-81.

14. Tzirakis K, Papaharilaou Y, Giordano D, Ekaterinaris J. Numerical investigation of biomagnetic fluids in circular ducts." International Journal for Numerical Methods in Biomedical Engineering. 2014; 30(3):297-317.

15. Misra JC, Shit GC. Effect of magnetic field on blood flow through an artery: a numerical model. Journal of Computational Technologies (Russia). 2007;12:4.

16. Probst $\mathrm{R}$, et al. Planar steering of a single ferrofluid drop by optimal minimum power dynamic feedback control of four electromagnets at a distance. Journal of Magnetism and Magnetic Materials. 2011;323(7):885-896.

17. Alexandru M, Mihaela M. Magnetic fieldflow interactions in drug delivery through an arterial system. Rev. Roumaine Sci.
Techn. Electrotech. et Energ. 2011;56(2): 199-208.

18. Wagh DK, Wagh SD. Blood flow considered as magnetic flow. Proceeding of Physiology of Fluid Dynamics III. 1992; 311-315.

19. Gupta AK. Finite element Galerkin's scheme for flow in blood vessels with magnetic effects. Int. J. Applied Systemtic Studies. 2009;3(2):283-293.

20. Haik Y, Pai V, Chen CJ. Biomagnetic fluid dynamics at interfaces'. Cambridge University Press, Cambridge. 1999;439452.

21. Berkovski B, Bashtovoy V. Magnetic fluids and applications handbook. Begell House inc., New York; 1996.

22. Furlani EP. Permanent Magnet and Electromechanical Device: Materials, Analysis and Applications". Academic, New York; 2001.

23. Bird RB, Armstrong RC, Hassager $\mathrm{O}$. Dynamics of polymeric fluids. Fluid Mechanics. Wiley: NY. 1987;1.

24. Ku David N. Blood flow in arteries. Annual Review of Fluid Mechanics. 1997;29(1): 399-434

25. Ferziger J, Peric M, Chap. 1Basic concepts of fluid flow, computational methods for fluid dynamics. 2002;1-12.

26. Blacher J, Asmar A, Djane S, London GM, Safar ME. Aortic pulse wave as a marker of cardiovascular risk in hypertensive patients". Hypertension. 1999;33:11111117.

27. Andreas Ö, Lucas da Silva FM. Analysis and design of biological materials and structures. Springer Science \& Business Media; 2012.

28. Salem S, Tuchin V. Trapping of magnetic nanoparticles in the blood stream under the influence of a magnetic field. Izv. Saratov Univ. (N. S.), Ser. Physics. 2020; 20(1):72-79.

Available:https://doi.org/10.18500/18173020-2020-20-1-72-79

(c) 2020 Salem and Tuchin; This is an Open Access article distributed under the terms of the Creative Commons Attribution License (http://creativecommons.org/licenses/by/4.0), which permits unrestricted use, distribution, and reproduction in any medium, provided the original work is properly cited.

Peer-review history:

The peer review history for this paper can be accessed here: http://www.sdiarticle4.com/review-history/60148 